\title{
Roles of Mannosylerythritol Lipid-B Components In Antimicrobial Activity Against Bovine Mastitis- Causing Staphylococcus Aureus
}

\section{Shinya Yamauchi}

Tohoku University Graduate School of Agricultural Science Faculty of Agriculture: Tohoku Daigaku Daigakuin Nogaku Kenkyuka Nogakubu

\section{Mutsumi Furukawa}

Tohoku University Graduate School of Agricultural Science Faculty of Agriculture: Tohoku Daigaku Daigakuin Nogaku Kenkyuka Nogakubu

\section{Akio Kawahara}

Toyobo Co., Ltd.

\section{Tomohiro Sugahara}

Toyobo Co., Ltd.

\section{Shuhei Yamamoto}

Toyobo Co., Ltd.

\section{Masao Kitabayashi}

Toyobo Co., Ltd

Atsushi Sogabe

Toyobo Co., Ltd.

\section{Eiji Hata}

National Agriculture and Food Research Organization: Nogyo Shokuhin Sangyo Gijutsu Sogo Kenkyu Kiko

\section{Kouichi Watanabe}

Tohoku University Graduate School of Agricultural Science Faculty of Agriculture: Tohoku Daigaku Daigakuin Nogaku Kenkyuka Nogakubu

\section{Hiroshi Yoneyama}

Tohoku University Graduate School of Agricultural Science Faculty of Agriculture: Tohoku Daigaku Daigakuin Nogaku Kenkyuka Nogakubu

\section{Hisashi Aso}

Tohoku University Graduate School of Agricultural Science Faculty of Agriculture: Tohoku Daigaku Daigakuin Nogaku Kenkyuka Nogakubu

\section{Tomonori Nochi ( $\nabla$ nochi@tohoku.ac.jp )}

Tohoku University Graduate School of Agricultural Science Faculty of Agriculture: Tohoku Daigaku Daigakuin Nogaku Kenkyuka Nogakubu https://orcid.org/0000-0003-1800-6972 


\section{Research Article}

Keywords: Mannosylerythritol lipid-B (MEL-B), bio-surfactant, S. aureus, mastitis, dairy cattle

Posted Date: June 15th, 2021

DOl: https://doi.org/10.21203/rs.3.rs-561655/v1

License: (c) (i) This work is licensed under a Creative Commons Attribution 4.0 International License. Read Full License

Version of Record: A version of this preprint was published at World Journal of Microbiology and Biotechnology on February 12th, 2022. See the published version at https://doi.org/10.1007/s11274-02203243-2. 


\title{
Roles of mannosylerythritol lipid-B components in antimicrobial activity against bovine mastitis-causing Staphylococcus aureus
}

\author{
Shinya Yamauchi ${ }^{1,2}$, Mutsumi Furukawa ${ }^{1,2}$, Akio Kawahara ${ }^{3}$, Tomohiro Sugahara ${ }^{3}$, \\ Shuhei Yamamoto ${ }^{3}$, Masao Kitabayashi ${ }^{4}$, Atsushi Sogabe ${ }^{3,4}$, \\ Eiji Hata ${ }^{5}$, Kouichi Watanabe ${ }^{1,2}$, Hiroshi Yoneyama ${ }^{1,6}$, \\ Hisashi Aso ${ }^{1,2,7}$, Tomonori Nochi ${ }^{1,2,7,8^{*}}$
}

${ }^{1}$ International Education and Research Center for Food and Agricultural Immunology, Graduate School of Agricultural Science, Tohoku University, Miyagi 980-8572, Japan

${ }^{2}$ Laboratory of Functional Morphology, Graduate School of Agricultural Science, Tohoku University, Miyagi 980-8572, Japan

${ }^{3}$ Toyobo Co., Ltd. Tsuruga Institute of Biotechnology, Fukui 914-0047, Japan

${ }^{4}$ Toyobo Co., Ltd. Biochemical Department, Osaka 530-8230, Japan

${ }^{5}$ Bacterial Pathogenesis Research Unit, Division of Bacterial and Parasitic Disease, National Institute of Animal Health, National Agriculture and Food Research Organization, Ibaraki 305-0856, Japan

${ }^{6}$ Laboratory of Animal Microbiology, Graduate School of Agricultural Science, Tohoku University, Miyagi 980-8572, Japan

${ }^{7}$ Laboratory of Animal Health Science, Graduate School of Agricultural Science, Tohoku University, Miyagi 980-8572, Japan

${ }^{8}$ Division of Mucosal Vaccines, International Research and Development Center for Mucosal Vaccines, The Institute of Medical Science, The University of Tokyo, Tokyo 108-8639, Japan ${ }^{*}$ Corresponding author

Correspondence: Tomonori Nochi, Ph.D.

International Education and Research Center for Food and Agricultural Immunology

Graduate School of Agricultural Science, Tohoku University

468-1 Aoba, Aramaki, Aoba-ku, Sendai, Miyagi 980-8572, Japan

Tel: +81-22-757-4312

Fax: +81-22-757-4315

Email: nochi@tohoku.ac.jp 


\begin{abstract}
Mannosylerythritol lipid-B (MEL-B), which comprises ester-bonded hydrophilic ME and hydrophobic fatty acids, is a bio-surfactant with various unique properties, including antimicrobial activity against most gram-positive bacteria. The gram-positive Staphylococcus aureus is a causative pathogen of dairy cattle mastitis, which results in considerable economic loss in dairy industry. Here, we demonstrate the efficacy of MEL-B as a disinfectant against bovine-derived S. aureus and elucidate a mechanism of action of MEL-B in inhibition of bacterial growth. The growth of bovine mastitis causative $S$. aureus BM1006 was inhibited when cultured with MEL-B above $10 \mathrm{ppm}$ (equivalent to $0.015 \mathrm{mM}$ ). The activity of MEL-B required fatty acids (i.e., caprylic and myristoleic acids) as ME, the component of MEL-B lacking fatty acids, did not inhibit the growth of $S$. aureus even at high concentrations. Importantly, ME-bound fatty acids effectively inhibited the growth of $S$. aureus when compared with free fatty acids. Specifically, the concentrations of ME-bound fatty acids and free caprylic and myristoleic acids required to inhibit the growth of $S$. aureus were 0.015 , 10 , and $1 \mathrm{mM}$, respectively. The involvement of ME in the antimicrobial activity of MEL-B was confirmed by digestion of MEL-B with lipase, which dissociated ME and fatty acids. These results indicated that a mechanism of action of MEL-B in inhibiting the growth of $S$. aureus could be explained by the effective transporting of antimicrobial fatty acids to the bacterial surface via hydrophilic ME.
\end{abstract}

Key words Mannosylerythritol lipid-B (MEL-B), bio-surfactant, S. aureus, mastitis, dairy cattle 


\section{Introduction}

In the livestock industry, large amounts of antibiotics have been extensively used to cure domestic animals suffering from infectious diseases such as mastitis, diarrhea, and pneumonia (Berendsen et al. 2015; Kromker and Leimbach 2017). Livestock have been also administered various antibiotics as feed additives to gain body weight rapidly; however, most countries recently proposed to discontinue using antibiotics as feed additives because of multiple safety concerns (Maron et al. 2013). The most serious issue caused by excess use of antibiotics is the development of antimicrobial resistant bacteria (AMR) that may then infect human beings (Graham et al. 2009; Lee 2003). The number of human deaths predicted in 2050 due to AMR infection has been estimated to be over 10 million if no actions are taken at the current time (de Kraker et al. 2016). Considering that contagious bacteria can spread globally, the idea of "One Health" that pursues the health of animals, humans, and the environment without the use of excess antibiotics has been widely accepted (Collignon 2015; McEwen and Collignon 2018).

Mastitis is caused primarily by infection with pathogens in the udder of mammals such as dairy cattle (Schrick et al. 2001). Bovine mastitis can be divided into two types based on the classification of causative bacteria. One type is caused environmentally (so-called environmental mastitis) by infection of bacteria that are commonly found in farms, such as environmental streptococci and Escherichia coli. The other type is infectious mastitis, which is mostly caused by Staphylococcus aureus (Hospido and Sonesson 2005). Of note, methicillin-resistant S. aureus (MRSA), a well-known AMR, has been frequently isolated from milk of dairy cattle with infectious mastitis (Vanderhaeghen et al. 2010). S. aureus forms a biofilm to facilitate infection and may survive latently in target cells, such as macrophages, by escaping from the host immune machinery (Scherr et al. 2015; Thurlow et al. 2011). Intracellular S. aureus is believed to account for why dairy cattle with subclinical mastitis cause large economical loss due to the reduction of milk production 
(Almeida et al. 1996; Sinha et al. 2014). Therefore, the development of novel strategies that can prevent infection by S. aureus in dairy cattle without using antibiotics not only contributes to "One health," especially in suppressing the outbreak of AMRs such as MRSA, but also facilitates reducing the economic loss caused by both clinical and subclinical mastitis (Sinha et al. 2014).

Mannosylerythritol lipid B (MEL-B) is a glycolipid bio-surfactant produced by the cultivation of Pseudozyma tsukubaensis (NBRC1940) in the presence of olive oil (Fukuoka et al. 2008). MEL-B is composed of hydrophilic 4-O- $\beta$-D-mannopyranosyl-D-erythritol (ME) and two types of hydrophobic fatty acids (e.g., caprylic acid and myristoleic acid in most cases) (Yamamoto et al. 2012). MEL-B possesses several biological characteristics because of the amphiphilic properties and is therefore attracting attention in various fields (Coelho et al. 2020). For instance, MEL-B has been commercialized in the cosmetic industry as a natural ingredient with a moisturizing effect, clearly indicating that there are no safety concerns (Yamamoto et al. 2012). Importantly, the properties of MEL-B include antibacterial activity against most gram-positive bacteria (Kitamoto et al. 1993); however, the mechanism of action is yet to be identified. Furthermore, the inhibitory effect of MEL-B on the growth of $S$. aureus strains derived from dairy cattle has not yet been investigated.

Here, we show that MEL-B inhibits the growth of a bovine mastitis causative $S$. aureus BM1006 and demonstrate that the hydrophilic ME acts as a delivery vehicle to transfer the antimicrobial fatty acids that affect bacterial growth. These results indicate that, as a bio-surfactant, MEL-B could be useful as a possible disinfectant capable of suppressing outbreaks of $S$. aureus in dairy farms. 


\section{Materials and Methods}

\section{MEL-B, ME, and fatty acids}

Pseudozyma tsukubaensis (NBRC1940) was cultured in production medium containing 10\% (v/v) olive oil, $0.1 \%(\mathrm{w} / \mathrm{v})$ yeast extract, $4 \%(\mathrm{w} / \mathrm{v})$ glucose, $0.3 \%(\mathrm{w} / \mathrm{v}) \mathrm{NaNO}_{3}, 0.03 \%(\mathrm{w} / \mathrm{v}) \mathrm{MgSO}_{4} \bullet 7$ $\mathrm{H}_{2} \mathrm{O}$, and $0.03 \%(\mathrm{w} / \mathrm{v}) \mathrm{KH}_{2} \mathrm{PO}_{4}$ at $25^{\circ} \mathrm{C}$ on a rotary shaker $(180 \mathrm{ppm})$ for 7 days to generate $\mathrm{MEL}-\mathrm{B}$, which was composed of ME and fatty acids, mainly caprylic (C8:0) and myristoleic (C14:1) acids. Purification of MEL-B from the culture medium of $P$. tsukubaensis was performed using highperformance liquid chromatography (HPLC) on a silica gel column. A portion of purified MEL-B was reacted with 4-N-chloroformylmethy-N-methyl(amino)-7-nitro2,1,3-benzoxadiazole, a fluorescent reagent with $\lambda \mathrm{ex}=470 \mathrm{~nm}$ and $\lambda \mathrm{em}=540 \mathrm{~nm}$, in dry acetone for $60 \mathrm{~min}$ to synthesize NBD-labeled MEL-B. ME was synthesized according to the previous study (Fukuoka et al. 2008).

\section{Bacterial strains and culture}

S. aureus BM1006 (MAFF913131) and E. coli JM109 (ATCC53323) were used in this study. Both bacteria were cultured overnight in Trypto-Soya (TS) broth (Nissui) at $37^{\circ} \mathrm{C}$ on a rotary shaker (120 ppm). Pre-cultured bacteria $(30 \mu \mathrm{L})$ were added to fresh TS broth $(3 \mathrm{~mL})$ containing MEL-B at various concentrations $(0,0.1,1,10,100$, and $1000 \mathrm{ppm})$ to address the effect of MEL-B on bacterial growth for $24 \mathrm{~h}$ at $37^{\circ} \mathrm{C}$. Additionally, S. aureus was cultured in TS broth containing ME at various concentrations $(0.1,1,10,100$, and $1000 \mathrm{ppm})$. S. aureus was also cultured for $5 \mathrm{~h}$ in the presence of either caprylic acid (1 and $10 \mathrm{mM})$ or myristoleic acid $(0.1$ and $1 \mathrm{mM})$. Survival of bacteria in the presence of either MEL-B, ME, caprylic, or myristoleic acid was determined by obtaining the numbers of colony-forming units (CFUs). Aliquots of bacterial broth were collected and seeded on TS agar plates (three replicates) after dilution with saline to obtain CFUs. 


\section{Binding analysis}

S. aureus BM1006 and E. coli JM109 were treated with 10 ppm of NBD-labeled MEL-B (NBDMEL-B) for $30 \mathrm{~min}$ at room temperature (RT). After washing three times with phosphate-buffered saline, bacteria were fixed in 4\% (w/v) of paraformaldehyde (Nacalai Tesque) for $30 \mathrm{~min}$ at RT and affixed on glass slides for fluorescence microscopy (BZ-9000, Keyence). Flow cytometry analysis (Accuri C6, DB) was also used to assess the mean fluorescence intensity of bacteria.

\section{Scanning electron microscopy analysis}

The effect of MEL-B on the surface structure of S. aureus BM1006 was investigated by scanning electron microscopy (SEM) analysis. Specifically, bacteria were cultured for $0.5,2,5$, and $24 \mathrm{~h}$ with or without $100 \mathrm{ppm}$ of MEL-B or were treated with $100 \mathrm{U} / \mathrm{mL}$ of lysostaphin (WAKO) for $30 \mathrm{~min}$ at RT. Bacteria were fixed in $2.5 \%(\mathrm{v} / \mathrm{v})$ of glutaraldehyde (Nacalai Tesque) for $1 \mathrm{~h}$ at $4^{\circ} \mathrm{C}$. After washing, bacteria were affixed to glass slides, coated with platinum and palladium, and analyzed using an SEM SU8000 (Hitachi) at $3.0 \mathrm{kV}$.

\section{HPLC analysis}

To quantify MEL-B contained in TS broth during the culture of $S$. aureus, HPLC was performed. Culture medium $(250 \mu \mathrm{L})$ collected after $0.5,2,5$, and $24 \mathrm{~h}$ of culturing was mixed with ethyl acetate $(500 \mu \mathrm{L})$ for $3 \mathrm{~min}$, and the supernatant was collected by centrifugation $\left(13,000 \mathrm{rpm}, 4^{\circ} \mathrm{C}\right.$, $3 \mathrm{~min})$. The process was repeated three times, and MEL-B was extracted by evaporating from the supernatant. Specifically, the quantification of the extracellular glycolipids was performed using the HPLC of the MEL-B extracts loaded onto a silica gel column (Inertsil SIL 100 A $5 \mu \mathrm{m}$, $4.6 \times 250 \mathrm{~mm}$; GL Science, Japan) using chloroform/methanol as the solvent system with a gradient flow (1 $\mathrm{mL} / \mathrm{min})$ controlled from 100:0 to 0:100. A low-temperature evaporate light-scattering 
detector (Shimadzu, Kyoto, Japan) was used for detecting MEL-B.

\section{Structural analysis of MEL-B on the antimicrobial activity}

MEL-B (1000 ppm) was treated with porcine pancreas-originated lipase (Sigma) at various concentrations $(0.2,2$, and $20 \mathrm{mg} / \mathrm{mL})$ for $1 \mathrm{~h}$ at $37^{\circ} \mathrm{C}$ at $1: 1$ ratio to cleave the ester bonds between ME and fatty acids. S. aureus was cultured with the reactant including MEL-B equivalent to $10 \mathrm{ppm}$ $(=0.015 \mathrm{mM})$ and also with a mixture of ME, caprylic acid, and myristoleic acid $(0.015 \mathrm{mM}$ each) to compare the efficacy between the mixture and intact MEL-B $(0.015 \mathrm{mM})$. After $5 \mathrm{~h}$, the survival of bacteria was determined by obtaining the CFU count.

\section{Statistics}

Statistical analyses were conducted using one-way analysis of variance (ANOVA) with the KruskalWallis test and two-way ANOVA with Tukey's multiple comparison test using Prism 7 (GraphPad). 


\section{Results}

MEL-B inhibits the growth of a bovine mastitis causative $S$. aureus strain BM1006

We first sought to demonstrate the efficacy of MEL-B as a possible disinfectant for use with dairy cattle in their breeding environment. Bovine milk-derived S. aureus BM1006, a bovine mastitis causative bacterium (Kiku et al. 2016), was cultured in vitro in the presence of MEL-B at 0 , $0.1,1,10,100$, and $1000 \mathrm{ppm}$, and the influence of MEL-B on the growth of S. aureus (via CFU counts) was monitored at $0.5,2,5$, and $24 \mathrm{~h}$. The growth of $S$. aureus was significantly delayed when cultured with MEL-B, and the minimum concentration of MEL-B required for complete inhibition of bacterial growth was $10 \mathrm{ppm}$ (Fig. 1). Consistent with a previous study that demonstrated the antimicrobial activity of MEL-B against most gram-positive (but not gram-negative) bacteria (Kitamoto et al. 1993), the growth of E. coli JM109, a representative gram-negative bacterium, was not significantly affected in the presence of MEL-B even at high concentrations (Fig. 2). These results support previous findings demonstrating the efficacy of MEL-B in inhibiting the growth of $S$. aureus (not E. coli) using bovine-derived S. aureus.

\section{MEL-B is stable during in vitro culture}

Although MEL-B possessed antimicrobial activity against $S$. aureus, the growth of $S$. aureus gradually continued during culture even in the presence of MEL-B at high concentrations. Therefore, we next addressed the condition of MEL-B during culture. Normal phase HPLC analysis showed that two peaks were present, one of which corresponded to intact MEL-B, whereas the other eluted at an earlier retention time corresponded unknown molecule(s) excluded by HPLC separation because of the low polarity (Fig. 3a). Importantly, the retention time of MEL-B did not change during culture with $S$. aureus, indicating that MEL-B is intact in our culture condition. In contrast, the first peak with a shorter retention time was slightly but obviously seen even in water (Fig. 3a). 
Furthermore, the amount of first peak increased during culture with S. aureus, resulting in a decrease in the proportion of MEL-B (Fig. 3b). These results suggest that hydrophobic molecule(s) produced by S. aureus may affect the antimicrobial effect of MEL-B in vitro.

\section{MEL-B associates with the cell surface of both $S$. aureus and $E$. coli}

To understand the molecular mechanism whereby MEL-B inhibits the growth of S. aureus, we labeled MEL-B with NBD and used this in a binding assay to confirm the association between MEL-B and S. aureus. The incubation of S. aureus with 10 ppm of NBD-labeled MEL-B (NBDMEL-B) made the bacterial surface clearly fluorescent under a microscope (Fig. 4a). Interestingly, $E$. coli JM109 treated with the same concentration of NBD-MEL-B displayed weak (but apparent) fluorescence despite the lack of inhibitory effect of MEL-B on the growth of E. coli (Fig. 4a). No fluorescence was seen in either untreated S. aureus or E. coli (Fig. 4a). We confirmed this result using flow cytometry analysis with $S$. aureus and E. coli, both of which were treated with NBDMEL-B at various concentrations $(0,0.1,1,10$, and $100 \mathrm{ppm})$. The analysis indicated that NBDMEL-B was associated with both S. aureus and E. coli in a dose-dependent manner (Fig. 4b). Furthermore, the fluorescence intensities observed in S. aureus were significantly higher than those in E. coli at NBD-MEL-B concentrations of 1 ppm or higher (Fig. 4c). These results suggest that the actions mediated by the binding of MEL-B to $S$. aureus (rather than simply the act of binding) may be necessary to inhibit bacterial growth.

\section{MEL-B does not damage the structure of $S$. aureus}

We then used SEM to chronologically examine the influence of MEL-B treatment on the structure of S. aureus during culture with MEL-B at relatively high concentration (100 ppm). Although MEL-B was anticipated to affect the surface structure of S. aureus by binding, no apparent 
differences could be seen in $S$. aureus during culture for $24 \mathrm{~h}$ with MEL-B when compared with $S$. aureus cultured without MEL-B (Fig. 5a). The typical shape of a grape-like cluster of S. aureus was observed regardless of MEL-B treatment (Fig. 5a). Moreover, these structures were completely different from those of damaged $S$. aureus treated with lysostaphin, which specifically disrupts the cell wall of $S$. aureus (Fig. 5b) (Watanakunakorn et al. 1971). These results suggest that the effect of MEL-B on the inhibition of the growth of $S$. aureus may not be due to the disruption of the overall bacterial structure.

Fatty acids, components of MEL-B, are involved in inhibiting the growth of $S$. aureus Since MEL-B is composed of mannopyranose-erythritol (ME), which has a hydrophilic property, and two different fatty acids [i.e., caprylic acid (C8:0) and myristoleic acid (C14:1)], which have hydrophobic properties (Yamamoto et al. 2012), we then identified the critical components of MEL-B that affect the growth of $S$. aureus. Supplementation of ME, obtained as an intermediate product in MEL-B synthesis, to $S$. aureus culture at various concentrations $(0,0.1,1,10,100$, and $1000 \mathrm{ppm}$ ) did not have any inhibitory effects, even at high concentrations (Fig. 6). Unfortunately, the binding capability of ME to the cell surface of $S$. aureus could not be investigated because of the technical difficulty of synthesizing fluorescence-conjugated ME; however, this result suggested that the hydrophobic fatty acid components of MEL-B may directly or indirectly inhibit the growth of $S$. aureus.

\section{ME acts as a delivery vehicle for caprylic acid and myristoleic acid to $S$. aureus}

To elucidate the mechanism of action of MEL-B on the growth inhibition of $S$. aureus, three related experiments were conducted to clarify the functions of ME and fatty acids. Both caprylic and myristoleic acids are known to possess antimicrobial activity against $S$. aureus (Kabara, 
1984). Therefore, we determined the minimum concentration of caprylic and myristoleic acids required to inhibit the growth of $S$. aureus. Since the efficacy of MEL-B in inhibiting the growth of S. aureus was clearly observed $5 \mathrm{~h}$ after culture (as shown in Fig. 1), S. aureus was cultured for $5 \mathrm{~h}$ in the presence of either caprylic acid ( 1 or $10 \mathrm{mM})$ or myristoleic acid $(0.1$ or $1 \mathrm{mM})$. There was a significant decrease in CFU when $10 \mathrm{mM}$ of caprylic acid or $1 \mathrm{mM}$ of myristoleic acid was added to the culture (Fig. 7a). We then compared the efficacy of MEL-B and a mixture of ME, caprylic acid, and myristoleic acid at the same concentration. The molecular weight of MEL-B composed of ME, caprylic acid, and myristoleic acid is $634 \mathrm{Da}$, and therefore, we used $10 \mathrm{ppm}$ (the minimum dose of MEL-B required to inhibit the growth of $S$. aureus), which is equivalent to $0.015 \mathrm{mM}$. However, a decrease in S. aureus CFU occurred when MEL-B at $0.015 \mathrm{mM}$ (but not the mixture of ME, caprylic acid, and myristoleic acid at $0.015 \mathrm{mM}$ each) (Fig. 7b). We then demonstrated the reduction of MEL-B efficacy by digestion of the ester bond that connects the ME with the two fatty acids. Specifically, $1000 \mathrm{ppm}$ of MEL that had been pretreated with lipase, which digests the ester bond, at various concentrations $(0.2,2$, and $20 \mathrm{mg} / \mathrm{mL})$ and $10 \mathrm{ppm}(=0.015 \mathrm{mM})$ equivalent of MEL-B was added in the culture medium of $S$. aureus. Importantly, MEL-B did not inhibit the growth of $S$. aureus when pretreated with the high concentration of lipase (Fig. 7c); the lipase itself did not affect the growth of S. aureus (Fig. 7c). These results indicated that the efficacy of MEL-B could be explained by the effective association of the antimicrobial caprylic and myristoleic acid components with the surface of $S$. aureus via the hydrophilic ME moiety. 


\section{Discussion}

Our study aimed to evaluate MEL-B as a possible disinfectant for use in the livestock industry to control the spread of $S$. aureus strains, including MRSA. We demonstrated that the growth of S. aureus BM1006, which belongs to the sequence type 352 that often causes mastitis in dairy cattle (Hata et al. 2010), is significantly inhibited when cultured in the presence of MEL-B in vitro. Notably, the pathogenicity of S. aureus BM1006 has been confirmed by in vivo infectious studies where mastitis was induced in dairy cattle when S. aureus BM1006 was inoculated into the udder (Nagasawa et al. 2018). A previous study has also shown that inflammatory chemokines (e.g., IL-8, CXCL6, and CCL2), which are involved in the recruitment of polymorphonuclear leukocytes into the udder, are abundantly secreted by mammary epithelial cells when stimulated with S. aureus BM1006 (Kiku et al. 2016). Given the virulence of S. aureus BM1006 as a causative bacteria of bovine mastitis, our results can provide insight into the development of a novel disinfectant to prevent the onset of mastitis in dairy cattle.

MEL-B had no effect on the growth of a representative gram-negative bacteria, E. coli JM109, in this study, which is consistent with previous results (Kitamoto et al. 1993). Our results also demonstrated that MEL-B bound to the cell surface of E. coli despite a lack of antimicrobial activity against the bacteria. Therefore, we hypothesized that further actions following the binding of MEL-B to the bacterial cell surface are required to inhibit the growth of gram-positive S. aureus (but not gram-negative E. coli). Recently, atomic force microscopy was used to demonstrate that the diameter of peptidoglycan pores can be up to $60 \mathrm{~nm}$ (Pasquina-Lemonche et al. 2020). Hence, MELB may diffuse freely into the cross-linked cell wall structure of gram-positive bacteria by penetrating the peptidoglycan interspaces and then interfering the cell membrane, where functional molecules (e.g., transporters and receptors) are involved in nutrient uptake and signal transduction are expressed. In contrast, lipopolysaccharide found in the outer membrane of gram-negative bacteria 
may interfere with the free diffusion of MEL-B, leaving the cell membrane intact in these bacteria even after binding.

One of the significant advances obtained in this study was to elucidate how MEL-B inhibits the growth of $S$. aureus. Specifically, the ME moiety plays an important role in effectively exerting antimicrobial activity of the caprylic and myristoleic acids against $S$. aureus. Nevertheless, S. aureus still grew for $24 \mathrm{~h}$ when cultured in the presence of MEL-B at high concentration. A key structural feature of MEL-B is the ester bonding that connects ME and the two fatty acids, which is important for exhibiting antimicrobial activity. Lipase produced by $S$. aureus during the culture was initially suspected as a factor that could reduce the antimicrobial activity of MEL-B. In this regard, pre-treatment of MEL-B with commercially available lipase from porcine pancreases reduced the antibacterial activity of MEL-B against $S$. aureus. However, the concentration of commercial lipase required to inhibit MEL-B activity was extremely high compared with that produced normally from S. aureus (Flanagan et al. 2016; Hu et al. 2012). Another possible hypothesis is that hydrophobic molecule(s) may affect the antimicrobial activity of MEL-B. HPLC analysis showed two major peaks corresponding to the MEL-B and unknown molecules(s) excluded by HPLC separation due to the low polarity (= high hydrophobicity). The peak with a shorter retention time was found even in water, and the amount increased during culture with $S$. aureus. These results suggest that the reduced antimicrobial activity of MEL-B against $S$. aureus during culture may be due to the effect of $S$. aureus-derived hydrophobic molecule(s) (but not impurities) in our culture condition. Therefore, further studies are needed to identify appropriate solvent conditions that maintain or increase the efficacy of MEL-B for practical application as a disinfectant for dairy cattle.

A recent study demonstrated that the apoptosis of $S$. aureus was induced by treatment with MEL-A (Shu et al. 2020). By contrast, in our study using MEL-B, there was no sign to demonstrate apparent cell death, such as membrane disruption, although the growth of $S$. aureus was successfully 
inhibited by the treatment of MEL-B. This discrepancy may be due to the structural difference between MEL-A and MEL-B. MELs are divided into four types (i.e., MEL-A, MEL-B, MEL-C, and MEL-D), based on the number and position of the acetyl group (Saika et al. 2018), which depends on the microorganism responsible for synthesis (Coelho et al. 2020). One of the notable characteristics of MEL-A, which differentiates this from MEL-B, MEL-C, or MEL-D, is that MELA possesses an additional acetyl group, resulting in a higher hydrophobicity compared with that of the others (Imura et al. 2007; Saika et al. 2018). Since we did not compare the antimicrobial activity between MEL-A and MEL-B, further studies should be conducted to determine which form of MEL is most appropriate in controlling the spread of S. aureus. Nevertheless, it should be emphasized that MEL-B has been extensively used in the cosmetic industry, where it is used to suppress skin perspiration and increase water content (Yamamoto et al. 2012). Although verification is required to determine the use of MEL-B as a disinfectant for controlling mastitis caused by $S$. aureus, the safe use of MEL-B as a moisturizer for skin care should be positively appreciated for potential application in the dairy industry.

In conclusion, we elucidated the mechanism of action of MEL-B, composed of ME and antimicrobial fatty acids, in inhibiting the growth of bovine-derived mastitis causative $S$. aureus. ME acts as a delivery vehicle for antimicrobial fatty acids. Therefore, the binding between ME and fatty acids via ester bond is essential for the effective transport of antimicrobial fatty acids that then associate with the surface of $S$. aureus. These results provide a novel insight into developing a disinfectant that could be used in farms to prevent dairy cattle from contracting mastitis.

Acknowledgments We thank Kozue Shoji and Kumiko Ito (Tohoku University) for their technical support to obtain SEM images. 
Author contribution ShiY and TN conceived and designed the research and wrote the manuscript. ShiY and MF performed experiments and analyzed the data. EH provided S. aureus BM1006 and supports experiments. ShuY, MK, AK, TS, and AS synthesized MEL-B. KW, HY, and HA support experiments.

Funding This study was funded in part by the Grants-in-Aid for Scientific Research (A) (18H03969) and the Core-to-Core Program (Advanced Research Networks) from Japan Society for the Promotion of Science (JSPS).

Data availability All data generated or analyzed during this study are included in this manuscript.

\section{Declarations}

Conflict of interest The authors declare no competing interests.

Ethical approval This study does not contain any experiments with human participants or animals. 


\section{References}

Almeida RA, Matthews KR, Cifrian E, Guidry AJ, Oliver SP (1996) Staphylococcus aureus invasion of bovine mammary epithelial cells. J Dairy Sci 79(6):1021-1026

Berendsen BJ, Wegh RS, Memelink J, Zuidema T, Stolker LA (2015) The analysis of animal faeces as a tool to monitor antibiotic usage. Talanta 132:258-268

Coelho ALS, Feuser PE, Carciofi BAM, de Andrade CJ, de Oliveira D (2020) Mannosylerythritol lipids: antimicrobial and biomedical properties. Appl Microbiol Biotechnol 104(6):22972318

Collignon P (2015) Antibiotic resistance: are we all doomed? Intern Med J 45(11):1109-1115

de Kraker ME, Stewardson AJ, Harbarth S (2016) Will 10 Million People Die a Year due to Antimicrobial Resistance by 2050? PLoS Med 13(11):e1002184

Flanagan JL, Khandekar N, Zhu H, Watanabe K, Markoulli M, Flanagan JT, Papas E (2016) Glycerol Monolaurate Inhibits Lipase Production by Clinical Ocular Isolates Without Affecting Bacterial Cell Viability. Invest Ophthalmol Vis Sci 57(2):544-550

Fukuoka T, Morita T, Konishi M, Imura T, Kitamoto D (2008) A basidiomycetous yeast, Pseudozyma tsukubaensis, efficiently produces a novel glycolipid biosurfactant. The identification of a new diastereomer of mannosylerythritol lipid-B. Carbohydr Res 343(3):555-560

Graham JP, Evans SL, Price LB, Silbergeld EK (2009) Fate of antimicrobial-resistant enterococci and staphylococci and resistance determinants in stored poultry litter. Environ Res 109(6):682-689

Hata E, Katsuda K, Kobayashi H, Uchida I, Tanaka K, Eguchi M (2010) Genetic variation among Staphylococcus aureus strains from bovine milk and their relevance to methicillin-resistant isolates from humans. J Clin Microbiol 48(6):2130-2139 
Hospido A, Sonesson U (2005) The environmental impact of mastitis: a case study of dairy herds. Sci Total Environ 343(1-3):71-82

Hu C, Xiong N, Zhang Y, Rayner S, Chen S (2012) Functional characterization of lipase in the pathogenesis of Staphylococcus aureus. Biochem Biophys Res Commun 419(4):617-620

Imura T, Hikosaka Y, Worakitkanchanakul W, Sakai H, Abe M, Konishi M, Minamikawa H, Kitamoto D (2007) Aqueous-phase behavior of natural glycolipid biosurfactant mannosylerythritol lipid A: sponge, cubic, and lamellar phases. Langmuir 23(4):1659-1663

Kabara JJ (1984) Antimicrobial agents derived from fatty acids. J Am Oil Chem Soc 61(2) 397-403

Kiku Y, Nagasawa Y, Tanabe F, Sugawara K, Watanabe A, Hata E, Ozawa T, Nakajima KI, Arai T, Hayashi T (2016) The cell wall component lipoteichoic acid of Staphylococcus aureus induces chemokine gene expression in bovine mammary epithelial cells. J Vet Med Sci 78(9):1505-1510

Kitamoto D, Yanagishita H, Shinbo T, Nakane T, Kamisawa C (1993) Surface active properties and antimicrobial activities of mannosylerythritol lipids as biosurfactant produced by Candida antarctica. J Biotech 29(1-2) 91-96

Kromker V, Leimbach S (2017) Mastitis treatment-Reduction in antibiotic usage in dairy cows. Reprod Domest Anim 52 Suppl 3:21-29

Lee JH (2003) Methicillin (Oxacillin)-resistant Staphylococcus aureus strains isolated from major food animals and their potential transmission to humans. Appl Environ Microbiol 69(11):6489-6494

Maron DF, Smith TJ, Nachman KE (2013) Restrictions on antimicrobial use in food animal production: an international regulatory and economic survey. Global Health 9:48 McEwen SA, Collignon PJ (2018) Antimicrobial Resistance: a One Health Perspective. Microbiol Spectr 6(2) 
Nagasawa Y, Kiku Y, Sugawara K, Tanabe F, Hayashi T (2018) Exfoliation rate of mammary epithelial cells in milk on bovine mastitis caused by Staphylococcus aureus is associated with bacterial load. Anim Sci J 89(1):259-266

Pasquina-Lemonche L, Burns J, Turner RD, Kumar S, Tank R, Mullin N, Wilson JS, Chakrabarti B, Bullough PA, Foster SJ, Hobbs JK (2020) The architecture of the Gram-positive bacterial cell wall. Nature 582(7811):294-297

Saika A, Koike H, Fukuoka T, Morita T (2018) Tailor-made mannosylerythritol lipids: current state and perspectives. Appl Microbiol Biotechnol 102(16):6877-6884

Scherr TD, Hanke ML, Huang O, James DB, Horswill AR, Bayles KW, Fey PD, Torres VJ, Kielian T (2015) Staphylococcus aureus Biofilms Induce Macrophage Dysfunction Through Leukocidin AB and Alpha-Toxin. mBio 6(4)

Schrick FN, Hockett ME, Saxton AM, Lewis MJ, Dowlen HH, Oliver SP (2001) Influence of subclinical mastitis during early lactation on reproductive parameters. J Dairy Sci 84(6):1407-1412

Shu Q, Wei T, Lu H, Niu Y, Chen Q (2020) Mannosylerythritol lipids: dual inhibitory modes against Staphylococcus aureus through membrane-mediated apoptosis and biofilm disruption. Appl Microbiol Biotechnol 104(11):5053-5064

Sinha MK, Thombare NN, Mondal B (2014) Subclinical mastitis in dairy animals: incidence, economics, and predisposing factors. ScientificWorldJournal 2014:523984

Thurlow LR, Hanke ML, Fritz T, Angle A, Aldrich A, Williams SH, Engebretsen IL, Bayles KW, Horswill AR, Kielian T (2011) Staphylococcus aureus biofilms prevent macrophage phagocytosis and attenuate inflammation in vivo. J Immunol 186(11):6585-6596

Vanderhaeghen W, Cerpentier T, Adriaensen C, Vicca J, Hermans K, Butaye P (2010) Methicillinresistant Staphylococcus aureus (MRSA) ST398 associated with clinical and subclinical 
mastitis in Belgian cows. Vet Microbiol 144(1-2):166-171

Watanakunakorn C, Fass RJ, Klainer AS, Hamburger M (1971) Light and scanning-beam electron microscopy of wall-defective Staphylococcus aureus induced by lysostaphin. Infect Immun $4(1): 73-78$

Yamamoto S, Morita T, Fukuoka T, Imura T, Yanagidani S, Sogabe A, Kitamoto D, Kitagawa M (2012) The moisturizing effects of glycolipid biosurfactants, mannosylerythritol lipids, on human skin. J Oleo Sci 61(7):407-412 


\section{Figure legends}

Fig. 1 MEL-B inhibits the growth of Staphylococcus aureus in vitro. Bovine-derived S. aureus BM1006 was cultured in the presence of MEL-B at 0, 0.1, 1, 10, 100, or $1000 \mathrm{ppm}$ for $0.5,2,5$, and $24 \mathrm{~h}$. The effect of MEL-B on the growth of S. aureus was assessed by counting colony-forming units (CFUs). Growth was significantly delayed when cultured with MEL-B above 10 ppm. Experiments were performed in triplicate, and the mean \pm standard error of the mean obtained from representative data is shown. Statistical analyses were conducted using two-way ANOVA with Tukey's multiple comparison test. $* * * * p<0.0001$

Fig. 2 MEL-B does not inhibit the growth of Escherichia coli in vitro. E. coli JM109, a representative of gram-negative bacteria, was cultured in the presence of MEL-B at $0,0.1,1,10$, 100 , or $1000 \mathrm{ppm}$ for $0.5,2,5$, and $24 \mathrm{~h}$. The effect of MEL-B on the growth of $E$. coli was assessed by counting colony-forming units (CFUs). MEL-B did not affect the growth of E. coli even at high concentrations. Experiments were performed in triplicate, and the mean \pm standard error of the mean obtained from representative data is shown. Statistical analyses were conducted using two-way ANOVA with Tukey's multiple comparison test

Fig. 3 MEL-B is stable during culture in TS broth. a $S$. aureus BM1006 was cultured in the presence of MEL-B at $1000 \mathrm{ppm}$ for $0.5,2,5$, and $24 \mathrm{~h}$. HPLC retention times of MEL-B in culture media were compared with those of fresh MEL-B undiluted or diluted with water or TS broth. In addition to the typical peak corresponding to MEL-B, another peak with a shorter retention time was detected when MEL-B was dissolved in water or TS broth, and the ratio of peak with a shorter retention time increased during culturing. b The proportion of the original peak corresponding to intact MEL-B was calculated. Statistical analyses were conducted using one-way ANOVA with the Kruskal-Wallis 
test. ${ }^{*} p<0.05, * * p<0.01$

Fig. 4 MEL-B binds to the surface of both $S$. aureus BM1006 and E. coli JM109 with different binding affinities. a Fluorescence signals were detected from $S$. aureus and E. coli when bacteria were incubated for 30 min in vitro with MEL-B that was pre-conjugated with a fluorescence molecule NBD (NBD-MEL-B). No fluorescence signals were seen from untreated S. aureus or E. coli. b Flow cytometric analysis at $0,0.1,1,10$, or 100 ppm of MEL-B showed that NBD-MEL-B associated with $S$. aureus and E. coli in a dose-dependent manner. c Mean fluorescence intensity (MFI) obtained from S. aureus was higher than that obtained from E. coli when bacteria were individually incubated with NBD-MEL-B above 1 ppm. Experiments were performed in triplicate. The representative data were shown in histograms and all MFIs obtained from three experiments were summarized in a dot graph. Statistical analyses were conducted using $t$-test. $* * * p<0.001$, $* * * * p<0.0001$

Fig. 5 MEL-B does not disrupt the surface structure of S. aureus BM1006. a Scanning electron microscopic (SEM) analysis showed that the morphology of S. aureus did not change through in vitro culture in the presence of a relatively high concentration (100 ppm) of MEL-B. b S. aureus was treated with $100 \mathrm{U} / \mathrm{mL}$ of lysostaphin to confirm the disruption of the bacterial structure as a control in the SEM analysis. Scale bar $=1 \mu \mathrm{m}$

Fig. 6 ME, a component of MEL-B, does not inhibit the growth of S. aureus BM1006 in vitro. S. aureus was cultured in the presence of ME at $0,0.1,1,10,100$, or $1000 \mathrm{ppm}$ for $0.5,2,5$, and $24 \mathrm{~h}$. The effect of ME on the growth of S. aureus was assessed by counting colony-forming units (CFUs). ME did not affect the growth of S. aureus even at high concentrations. Experiments were performed 
in triplicate, and the mean \pm standard error of the mean obtained from representative data is shown. Statistical analyses were conducted using two-way ANOVA with Tukey's multiple comparison test

Fig. 7 Modification of ME with two fatty acids is essential for effectively inhibiting the growth of $S$. aureus BM1006. a Caprylic acid (C8:0) and myristoleic acid (C14:1), both of which associate with ME, showed clear antimicrobial activity against $S$. aureus when cultured for $5 \mathrm{~h}$ at 10 and $1 \mathrm{mM}$, respectively. b A mixture of ME with caprylic and myristoleic acid, which were adjusted to the same concentration as MEL-B $(10 \mathrm{ppm}=0.015 \mathrm{mM})$, did not inhibit the growth of S. aureus. c Pretreatment of MEL-B with a high concentration of lipase to digest the ester bond between the two fatty acids and ME reduced the antibacterial activity against $S$. aureus. Experiments were performed in triplicate, and the mean \pm standard error of the mean obtained from representative data is shown. Statistical analyses were conducted using one-way ANOVA with the Kruskal-Wallis test. ${ }^{* *} p<$ $0.01,{ }^{* * *} p<0.001,{ }^{* * * *} p<0.0001$ 


\section{Figures}

\section{S. aureus BM1006}

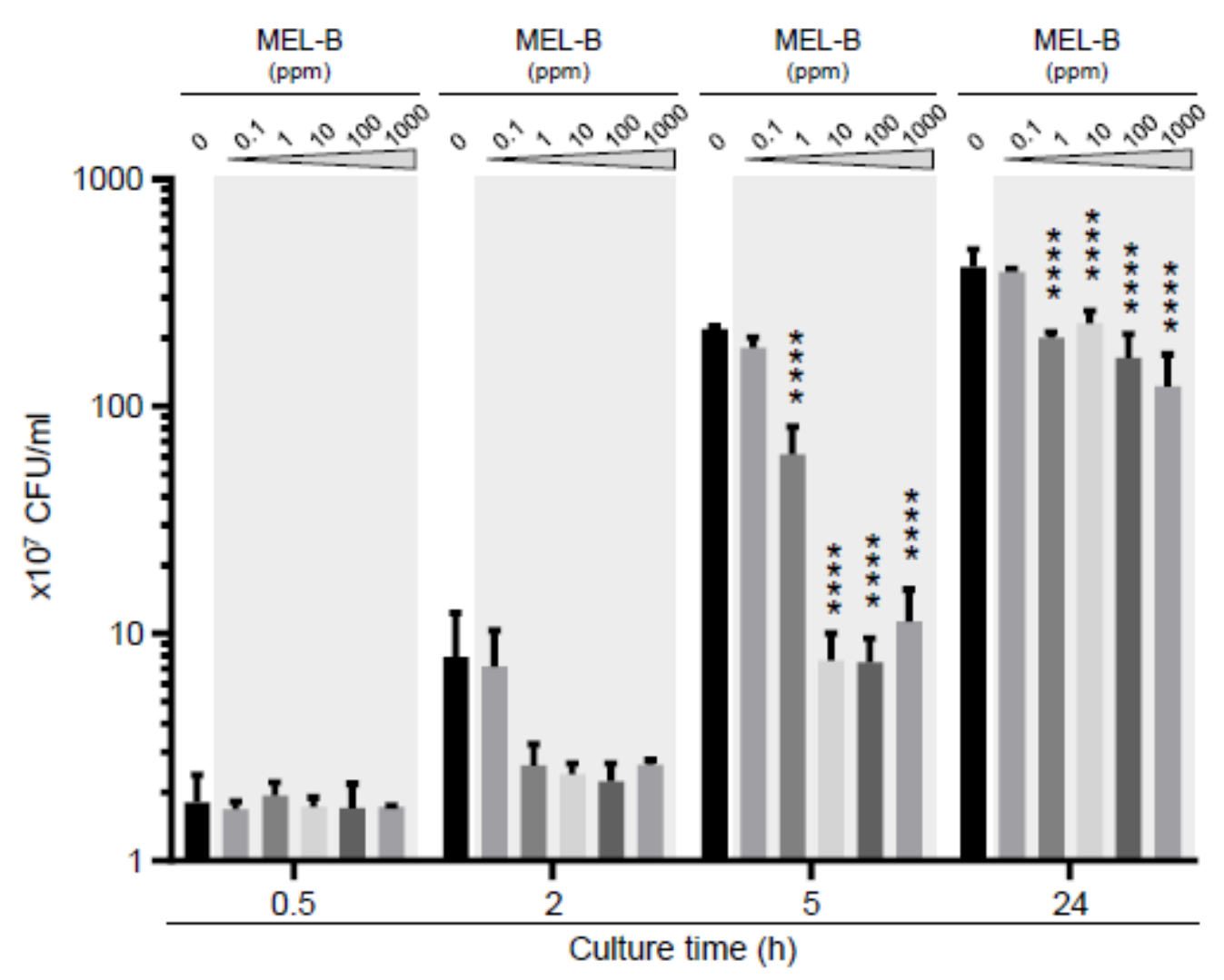

Figure 1

MEL-B inhibits the growth of Staphylococcus aureus in vitro. Bovine-derived S. aureus BM1006 was cultured in the presence of MEL-B at $0,0.1,1,10,100$, or $1000 \mathrm{ppm}$ for $0.5,2,5$, and $24 \mathrm{~h}$. The effect of MEL-B on the growth of $\mathrm{S}$. aureus was assessed by counting colony-forming units (CFUs). Growth was significantly delayed when cultured with MEL-B above $10 \mathrm{ppm}$. Experiments were performed in triplicate, and the mean \pm standard error of the mean obtained from representative data is shown. Statistical analyses were conducted using two-way ANOVA with Tukey's multiple comparison test. ${ }^{\star \star \star \star} p<0.0001$ 


\section{E. coli JM109}

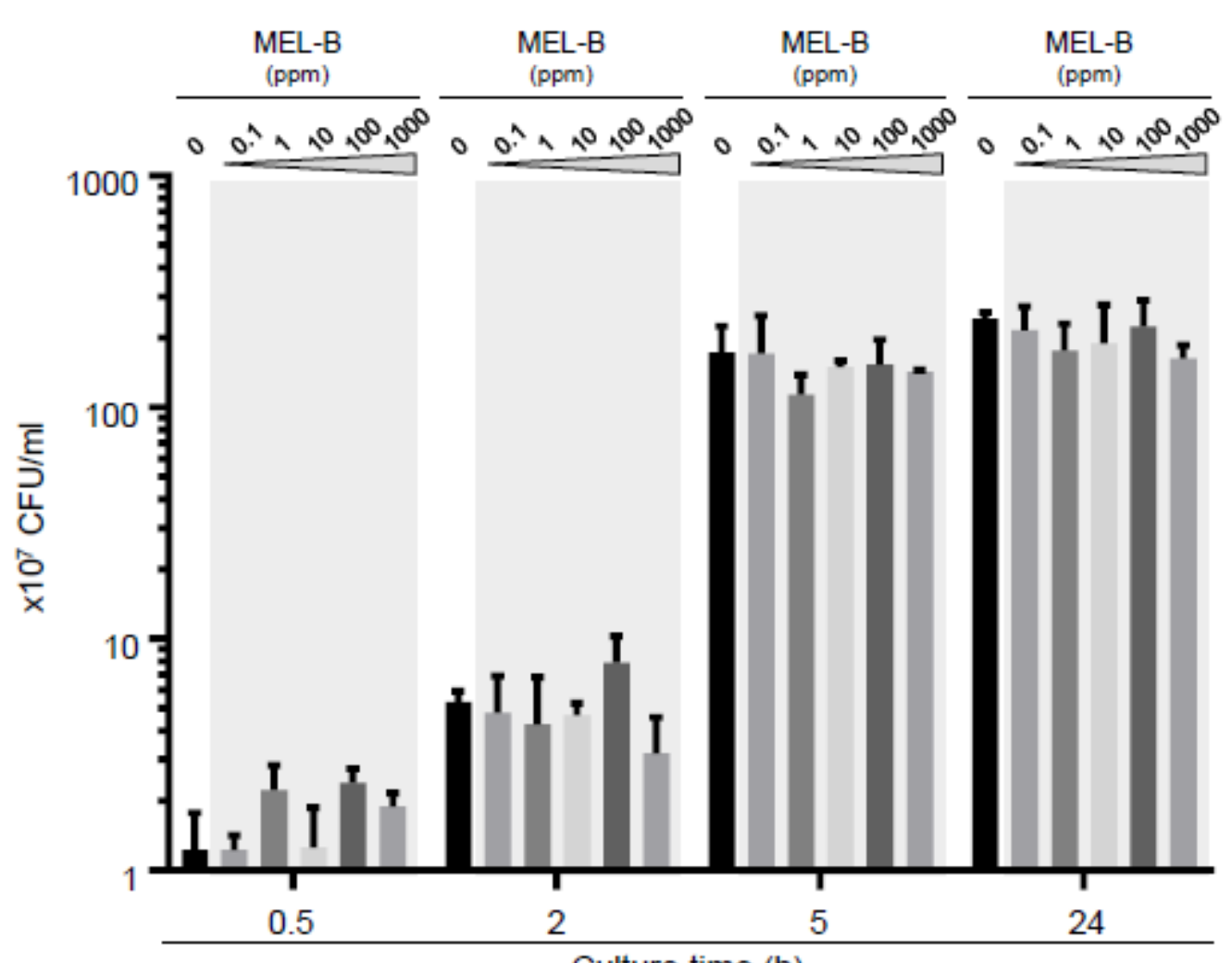

Figure 2

MEL-B does not inhibit the growth of Escherichia coli in vitro. E. coli JM109, a representative of gramnegative bacteria, was cultured in the presence of MEL-B at $0,0.1,1,10,100$, or $1000 \mathrm{ppm}$ for $0.5,2,5$, and $24 \mathrm{~h}$. The effect of MEL-B on the growth of E. coli was assessed by counting colony-forming units (CFUs). MEL-B did not affect the growth of E. coli even at high concentrations. Experiments were performed in triplicate, and the mean \pm standard error of the mean obtained from representative data is shown. Statistical analyses were conducted using two-way ANOVA with Tukey's multiple comparison test 
a

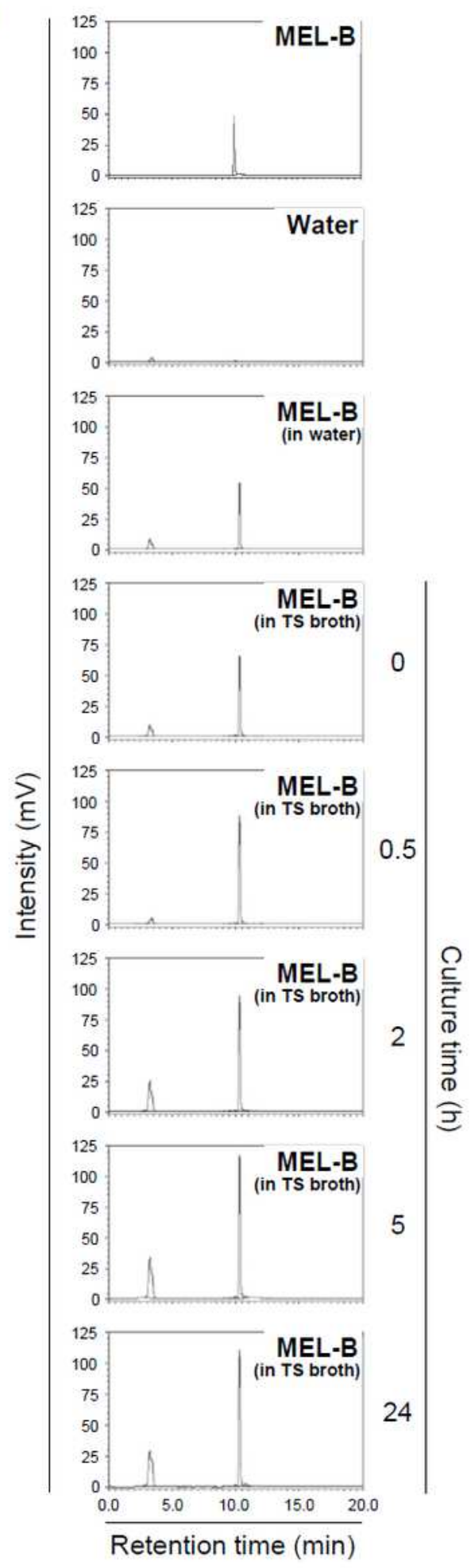

b

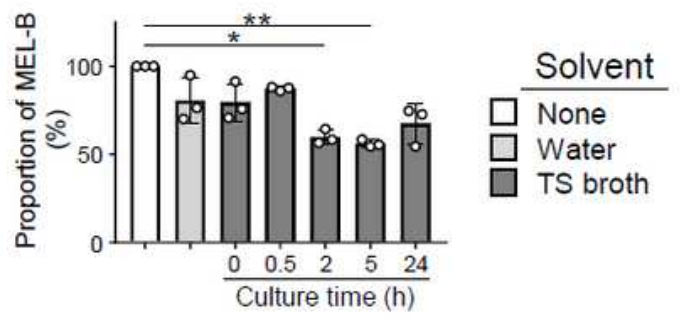

\section{Figure 3}

MEL-B is stable during culture in TS broth. a S. aureus BM1006 was cultured in the presence of MEL-B at 1000 ppm for $0.5,2,5$, and $24 \mathrm{~h}$. HPLC retention times of MEL-B in culture media were compared with those of fresh MEL-B undiluted or diluted with water or TS broth. In addition to the typical peak corresponding to MEL-B, another peak with a shorter retention time was detected when MEL-B was dissolved in water or TS broth, and the ratio of peak with a shorter retention time increased during 
culturing. b The proportion of the original peak corresponding to intact MEL-B was calculated. Statistical analyses were conducted using one-way ANOVA with the Kruskal-Wallis test. ${ }^{*} p<0.05,{ }^{*} p<0.01$

a

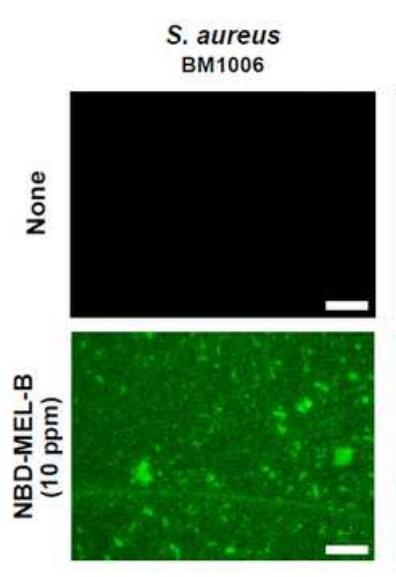

c
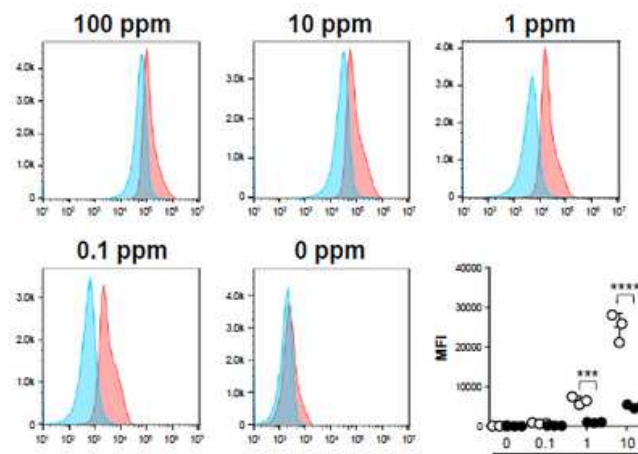

E. coli
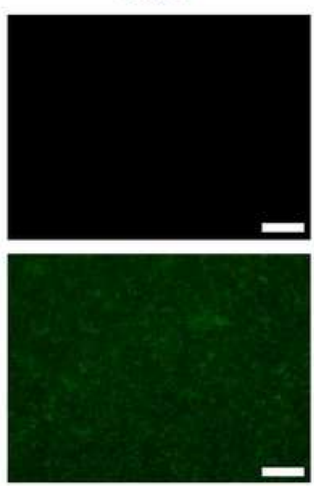

b
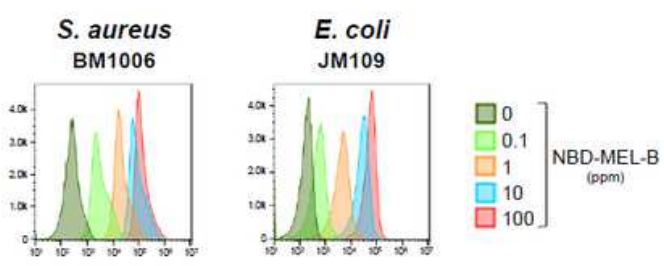

\section{Figure 4}

MEL-B binds to the surface of both S. aureus BM1006 and E. coli JM109 with different binding affinities. a Fluorescence signals were detected from $\mathrm{S}$. aureus and $\mathrm{E}$. coli when bacteria were incubated for $30 \mathrm{~min}$ in vitro with MEL-B that was pre-conjugated with a fluorescence molecule NBD (NBD-MEL-B). No fluorescence signals were seen from untreated $S$. aureus or E. coli. b Flow cytometric analysis at $0,0.1,1$, 10, or 100 ppm of MEL-B showed that NBD-MEL-B associated with S. aureus and E. coli in a dosedependent manner. c Mean fluorescence intensity (MFI) obtained from S. aureus was higher than that obtained from E. coli when bacteria were individually incubated with NBD-MEL-B above 1 ppm. Experiments were performed in triplicate. The representative data were shown in histograms and all MFIs obtained from three experiments were summarized in a dot graph. Statistical analyses were conducted

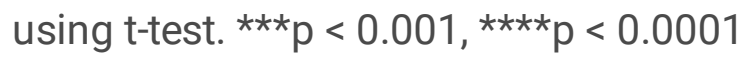




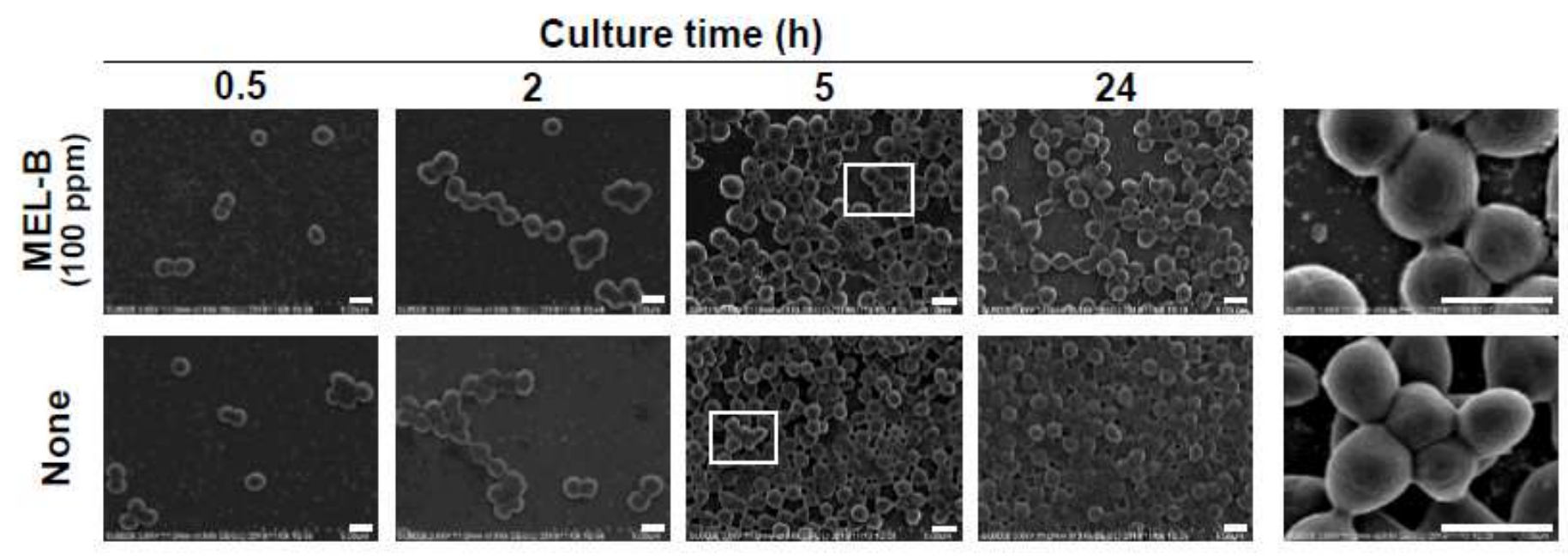

b

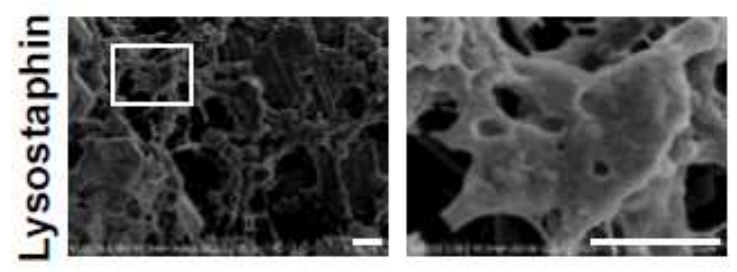

Figure 5

MEL-B does not disrupt the surface structure of S. aureus BM1006. a Scanning electron microscopic (SEM) analysis showed that the morphology of $\mathrm{S}$. aureus did not change through in vitro culture in the presence of a relatively high concentration (100 ppm) of MEL-B. b S. aureus was treated with $100 \mathrm{U} / \mathrm{mL}$ of lysostaphin to confirm the disruption of the bacterial structure as a control in the SEM analysis. Scale bar $=1 \mu \mathrm{m}$ 


\section{S. aureus BM1006}

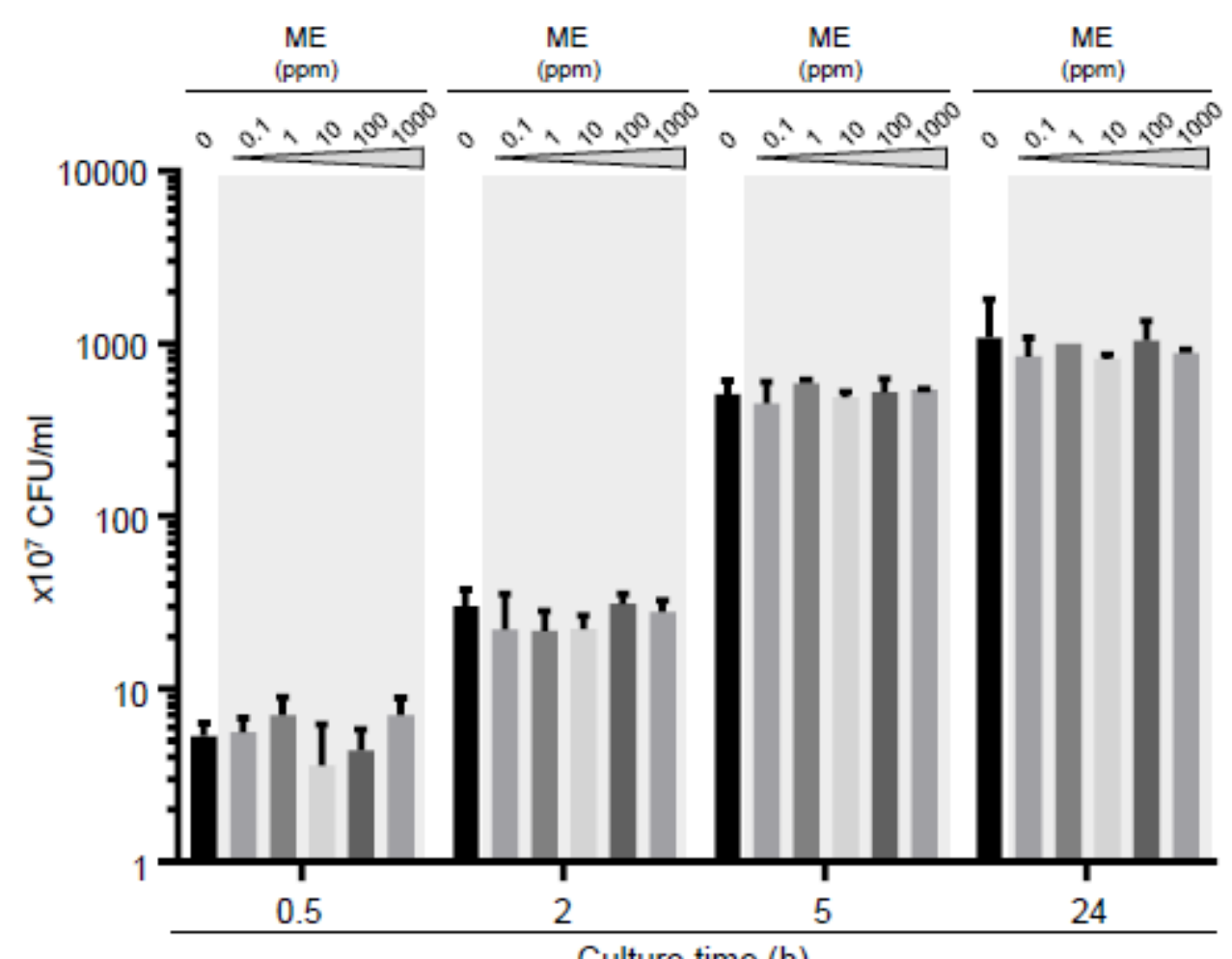

Figure 6

$\mathrm{ME}$, a component of MEL-B, does not inhibit the growth of S. aureus BM1006 in vitro. S. aureus was cultured in the presence of ME at $0,0.1,1,10,100$, or $1000 \mathrm{ppm}$ for $0.5,2,5$, and $24 \mathrm{~h}$. The effect of ME on the growth of $\mathrm{S}$. aureus was assessed by counting colony-forming units (CFUs). ME did not affect the growth of S. aureus even at high concentrations. Experiments were performed in triplicate, and the mean \pm standard error of the mean obtained from representative data is shown. Statistical analyses were conducted using two-way ANOVA with Tukey's multiple comparison test 
a

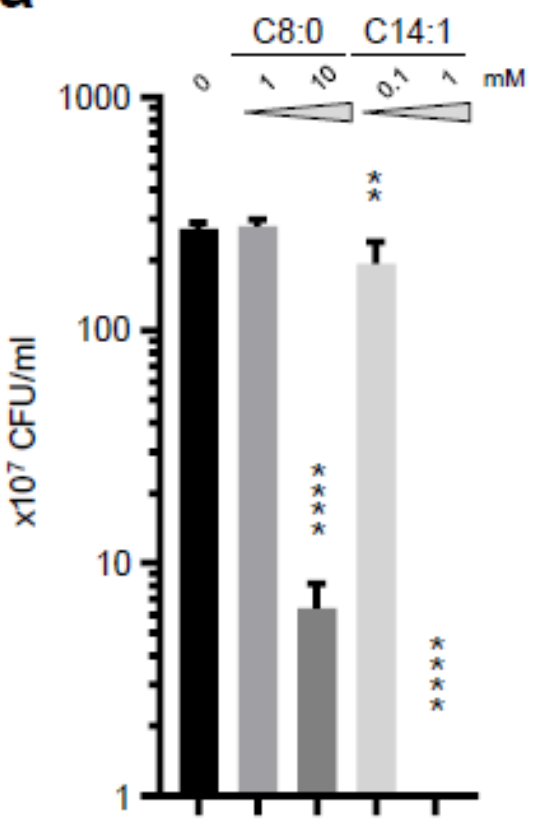

b

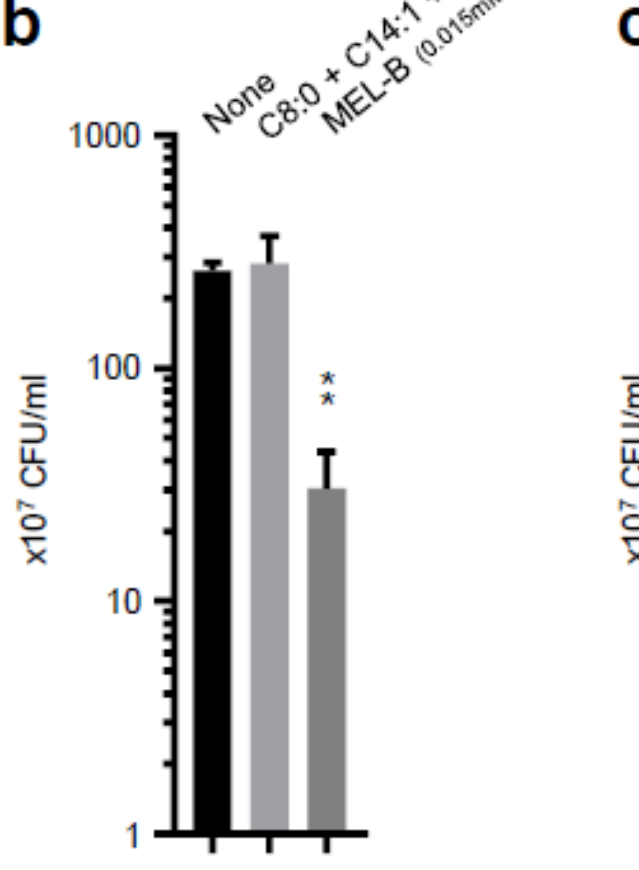

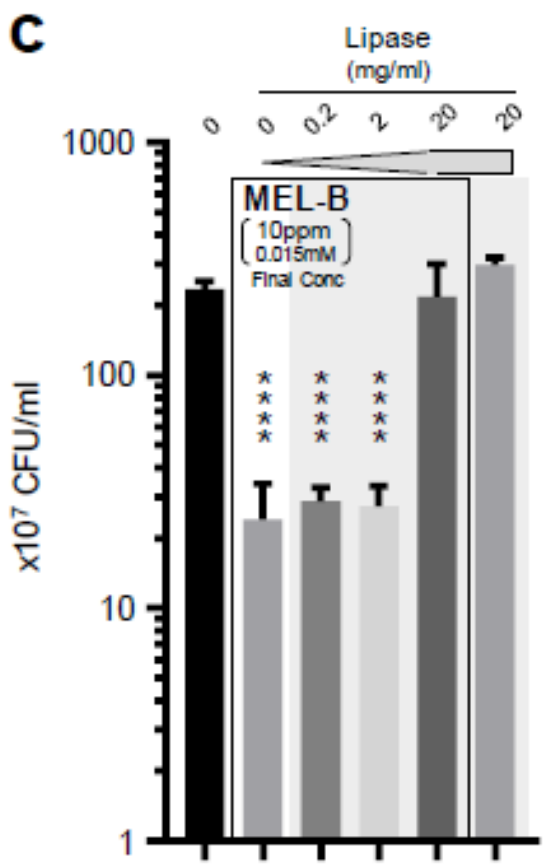

\section{Figure 7}

Modification of ME with two fatty acids is essential for effectively inhibiting the growth of S. aureus BM1006. a Caprylic acid (C8:0) and myristoleic acid (C14:1), both of which associate with ME, showed clear antimicrobial activity against $\mathrm{S}$. aureus when cultured for $5 \mathrm{~h}$ at 10 and $1 \mathrm{mM}$, respectively. $\mathrm{b} A$ mixture of ME with caprylic and myristoleic acid, which were adjusted to the same concentration as MEL$B(10 \mathrm{ppm}=0.015 \mathrm{mM})$, did not inhibit the growth of S. aureus. c Pre-treatment of MEL-B with a high concentration of lipase to digest the ester bond between the two fatty acids and ME reduced the antibacterial activity against $\mathrm{S}$. aureus. Experiments were performed in triplicate, and the mean \pm standard error of the mean obtained from representative data is shown. Statistical analyses were conducted using one-way ANOVA with the Kruskal-Wallis test. ${ }^{* \star} p<0.01,{ }^{\star \star \star} p<0.001,{ }^{\star \star \star \star} p<0.0001$ 